\title{
Antiplaque and Antigingivitis Effectiveness of Tea Tree Oil and Chlorine Dioxide Mouthwashes among Young Adults: A Randomized Controlled Trial
}

\author{
Aarya N Bharadwaj ${ }^{1}$, Vijayalakshmi Byatappa ${ }^{2}$, Rekha Raju$^{3}$, Rekha Umakanth ${ }^{4}$, Prajila Alayadan ${ }^{5}$
}

\begin{abstract}
Aim: Gingivitis is a common oral condition that can lead to severe dental complications. Prior research has shown the antimicrobial efficacies of tea tree oil (TTO) and chlorine dioxide $\left(\mathrm{ClO}_{2}\right)$ against oral microorganisms. The aim of this study is to evaluate the effect of these mouthwashes against dental plaque accumulation and gingival inflammation among young adults.

Materials and methods: This study followed a single-center, parallel-group, double-blind, randomized controlled trial design and was conducted in Bengaluru among 60 participants aged 18-25 years. Participants were randomly allocated into one of the three study groups (chlorhexidine, TTO, chlorine dioxide) with 20 participants in each and used the respective mouthwash twice daily along with other oral hygiene practices. The modified Silness and Loe plaque index and the Loe and Silness gingival index were used to assess plaque accumulation and gingival inflammation at both times-baseline and follow-up (21 days). The paired $t$-test and nonparametric tests like Wilcoxon sign-rank test and Kruskal-Wallis test with post hoc Dunn test were used to assess the outcome measures. A $p$ value of $<0.05$ was considered statistically significant.

Results: A statistically significant reduction in mean gingival and plaque scores was observed in all three groups after 21 days. Tea tree oil and chlorhexidine were comparable in reducing plaque scores, whereas chlorhexidine was superior in reducing gingival scores.

Conclusion: Tea tree oil and chlorine dioxide were effective in reducing plaque accumulation and gingivitis and could be suitable alternatives for chlorhexidine.

Clinical significance: Mouthwashes are the most widely used chemical adjuncts to mechanical plaque control and adverse effects of chlorhexidine digluconate have limited its use in cases of gingivitis. Thus, among the alternatives available, there is a need for the product with minimum side effects and maximum efficacy and so this study evaluated the effect of TTO and chlorine dioxide mouth rinses and compared their effects with chlorhexidine against dental plaque accumulation and gingival inflammation among young adults.

Keywords: Chlorine dioxide, Dental plaque, Gingivitis, Mouthwashes, Oral hygiene, Tea tree oil.

World Journal of Dentistry (2020): 10.5005/jp-journals-10015-1765
\end{abstract}

\section{INTRODUCTION}

Good oral hygiene refers to the maintenance of a clean mouth that is free from disease and is usually achieved by regular and correct toothbrushing. The chief culprit in the etiopathogenesis of the two most common dental conditions, periodontal disease and dental caries, is the oral biofilm. ${ }^{1}$ Therefore, optimal plaque control that removes the biofilm plays an essential role in preventing and/or arresting gingival inflammation among other oral conditions. ${ }^{2}$

Mouthwash or oral rinse is the most widely used adjunct to routine toothbrushing. They are oral antiseptic solutions that function to reduce the microbial plaque. ${ }^{3}$ Extracts of many medicinal plants can reduce free radical species, possess antimicrobial and anti-inflammatory properties, and have been used for prevention of various oral infections. ${ }^{4}$ Tea tree oil (TTO) or Melaleuca alternifolia is derived from the paperbark tea tree belonging to the family Myrtaceae. Tea tree oil is mainly composed of 1,8-cineole, terpinene-4-ol, gamma-terpinene, and alpha-terpineol. Its range of antimicrobial activity is similar to chlorhexidine, only differing in its mechanisms of action. ${ }^{5,6}$ Thus, TTO has the potential to be a therapeutic agent in chronic gingivitis and periodontitisconditions that have both bacterial and inflammatory components, owing to its antibacterial, antifungal, and antiviral properties. ${ }^{5}$

Stabilized chlorine dioxide $\left(\mathrm{ClO}_{2}\right)$ is a potent, topical oral antiseptic available in the market with minimal side effects. A gas at room temperature, its relatively stable free radical species $\left(\mathrm{ClO}_{2}^{\circ}\right)$ is

\footnotetext{
${ }^{1-3}$ Department of Public Health Dentistry, VS Dental College and Hospital, Bengaluru, Karnataka, India

4,5 Department of Pharmaceutics, KLE College of Pharmacy, Bengaluru, Karnataka, India
}

Corresponding Author: Aarya N Bharadwaj, Department of Public Health Dentistry, VS Dental College and Hospital, Bengaluru, Karnataka, India, Phone: +918951112492, e-mail: bharadwaj.aarya@ gmail.com

How to cite this article: Bharadwaj AN, Byatappa V, Raju R, et al. Antiplaque and Antigingivitis Effectiveness of Tea Tree Oil and Chlorine Dioxide Mouthwashes among Young Adults: A Randomized Controlled Trial. World J Dent 2020;11(6):451-456.

Source of support: Nil

Conflict of interest: None

a broad-spectrum, powerful chemical bactericidal that functions by oxidative consumption of critical biomolecules. It disrupts transport of nutrients across the cell wall and its single electron reduction product (chlorite ion $\mathrm{ClO}_{2}^{-}$) functions as a reactive oxidant toward many electron-donating biomolecules (e.g., methionine, pyruvate, urate, cysteine) present in periodontopathogens. It is effective in reducing plaque accumulation and formation, and in treating halitosis and gingivitis. ${ }^{7,8}$ 
While chlorhexidine (CHX) digluconate, a bisbiguanide broadspectrum antimicrobial agent, has been extensively studied for plaque control, its local side effects like altered taste sensation, staining of teeth, and desquamation of oral mucosa have limited its long-term use. ${ }^{9}$ Therefore, this study aimed to evaluate the effectiveness of TTO and chlorine dioxide mouthwashes and to compare their effects with chlorhexidine digluconate on plaque accumulation and gingival inflammation among 18-25-year-old adults.

\section{Materials and Methods}

A randomized controlled trial following a double-blind, singlecenter, parallel-arm design was conducted between November and December 2019 in accordance with the CONSORT statement guidelines for these trials. The study was approved by the institutional ethics committee and participants provided written informed consent before enrolment. The study was conducted in full accordance with the World Medical Association Declaration of Helsinki.

\section{Study Participants and Eligibility}

The study was conducted among college students who were selected based on the following inclusion criteria: (a) 18-25 years of age; (b) plaque index score and gingival index score of $>1$ in $10 \%$ of the sites; and (c) willing to be a part of the study and provide consent to adhere to the schedule. The exclusion criteria were as follows: (a) participants undergoing orthodontic treatment or using a removable appliance; (b) allergies/systemic diseases; and (c) already using a mouth rinse. Eligible participants were divided into three groups: TTO mouthwash group, chlorine dioxide mouthwash group, and positive control chlorhexidine mouthwash group. A well-illuminated room on the college premises was provided by the authorities for examination during baseline and follow-up, which was conducted during the morning session on 2 days each, 21 days apart.

\section{Sample Size Estimation}

Based on a previous study, ${ }^{10}$ a reduction of 0.08 compared to active mouthwash was considered acceptable. A minimum of 20 participants per group were calculated to achieve $90 \%$ statistical power assuming 5\% alpha error and $15 \%$ expected withdrawal/ dropout rate.

\section{Assessment}

At baseline, all study participants were then evaluated for plaque and gingival inflammation according to the modified plaque index by Silness and Loe ${ }^{11}$ and modified gingival index by Loe and Silness. ${ }^{12}$ Plaque accumulation was assessed using mouth mirrors and dental explorers, without the use of a disclosing solution, while gingival index was recorded using mouth mirrors and periodontal probes. Those eligible then filled the questionnaire, were randomized, and followed up for 21 days.

\section{Mouthwash Preparation}

Commercially available $0.12 \%$ chlorhexidine digluconate (Guard-OR, Group Pharmaceuticals Ltd, India) and stabilized chlorine dioxide (Freshchlor, Group Pharmaceuticals Ltd, India) mouthwashes were used. Tea tree oil mouthwash was formulated and prepared at a pharmacy institution in the same city. The ingredients used included TTO $(0.5 \mathrm{~g})$, Milli-Q water q.s. $(100 \mathrm{~mL} / \mathrm{g})$, Tween-80
(2.5 g), propylene glycol (5 g), glycerin (5 g), and benzyl alcohol $(0.2 \mathrm{~g})$. Propylene glycol and glycerin were weighed separately and mixed. To this mixture, Tween- 80 was added next, followed by TTO (NATURALIS: Essence of Nature Tea tree essential oil, Bengaluru, India), 50\% of Milli-Q water, and benzyl alcohol. Last, water was added to increase the volume of the mixture to $100 \mathrm{~mL}$. No flavoring agent was added.

\section{Randomization and Administration of the Mouthwash}

Eligible participants were randomly assigned to one of the three groups using the lottery method by another person, who was not involved in the study. The principal investigator was blinded to the randomization process and was not aware of the assigned group during outcome evaluations to prevent bias. About $500 \mathrm{~mL}$ of each mouthwash was dispensed in identical bottles. Preassigned identification codes for each group, set by the person not involved in the study, were printed on the plain containers along with instructions to use them. Participants were instructed to use the respective mouthwashes twice daily, once after breakfast and once after dinner. Instructions for each rinse was given as well: $10 \mathrm{~mL}$ of mouthwash taken in the bottle cap was to be swished in the mouth for 30 seconds and then spit out. Participants were told to avoid eating, drinking, or rinsing their mouth for the next 30 minutes and were asked to continue their preexisting oral hygiene practices. At follow-up, after 21 days, the participants were evaluated for plaque accumulation and gingival inflammation using the same scoring criteria. Adherence to the study schedule was confirmed by checking the quantity of mouthwash remaining in the bottles. All assessments at baseline and follow-up were made by the principal investigator who was previously trained.

The primary outcome was the difference in mean changes of plaque index scores and gingival index scores from baseline till 21 days between the groups.

\section{Statistical Analysis}

Data normality was assessed using the Shapiro-Wilk test. The paired $t$-test and Wilcoxon matched-pair signed-ranks test were used to compare the mean plaque index score and mean gingival index score before and after the mouthwash use within each group, respectively. The differences in plaque index or gingival index between groups were evaluated using the Kruskal-Wallis test. The statistical software package SPSS (IBM SPSS Statistics for Windows, version 22, Armonk, NY: IBM Corp.) was used and level of statistical significance was set at $<0.05$.

\section{Results}

Of a total of 70 eligible participants, 60 agreed to participate and were randomized in three groups $(n=20)$ as shown in Flowchart 1. All participants attended all examinations. The mean age was 19.38 \pm 0.95 years and had a higher percentage of males $(n=65 \%)$ (Table 1).

All the study participants used toothbrushes and toothpaste to clean their teeth and more than half brushed their teeth and tongue once daily in a combination (vertical and horizontal) brushing motion. About $21.7 \%$ of the population who consumed tobacco smoked cigarettes while more than three-quarters followed a mixed diet and consumed sugary food frequently (Fig. 1). No statistically significant differences were observed among the participants in all three groups with regard to these. 
Flowchart 1: Flowchart of study participants. TTO, tea tree oil mouthwash group; $\mathrm{ClO}_{2}$, chlorine dioxide mouthwash group; $\mathrm{CHX}$, chlorhexidine mouthwash group

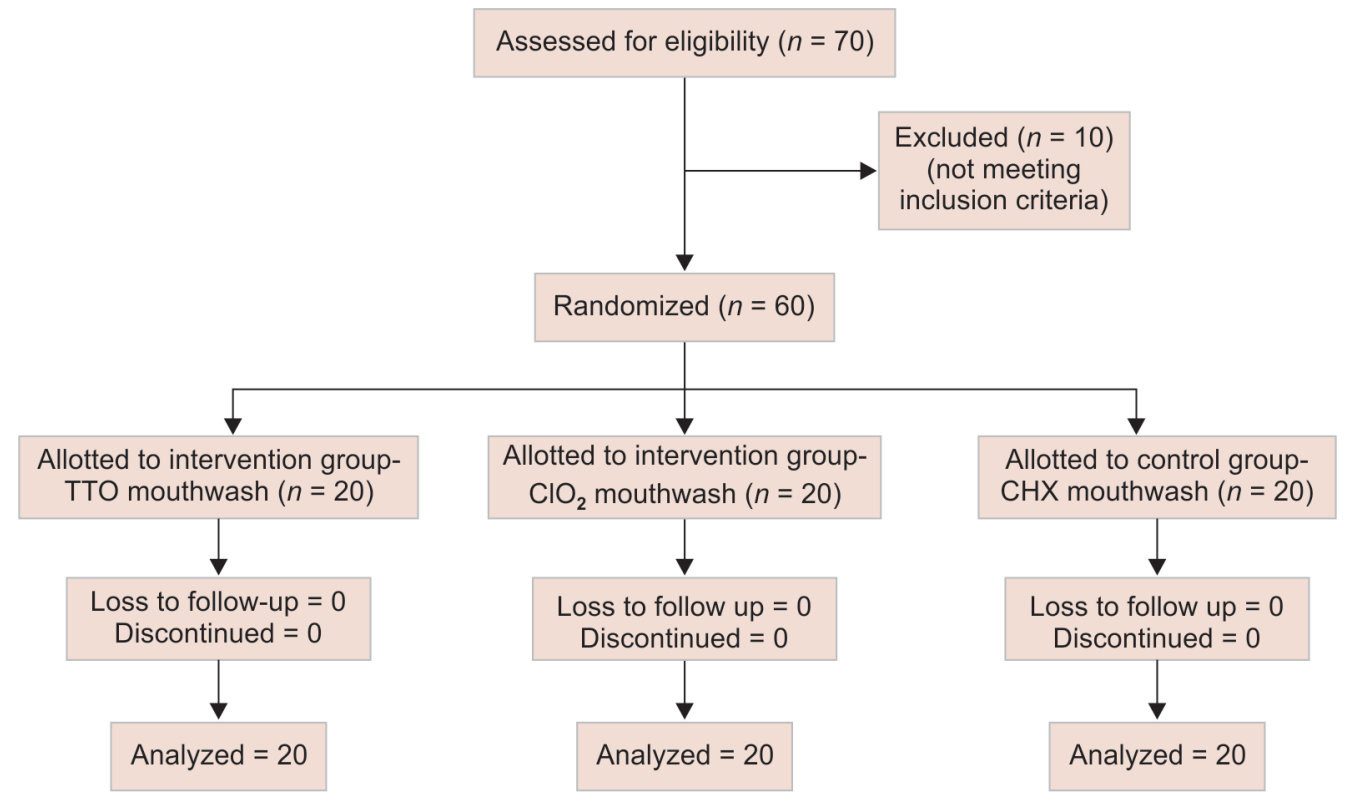

Table 1: Study participants distribution based on age and gender

\begin{tabular}{llllll}
\hline & & TTO $n(\%)$ & $\mathrm{ClO}_{2} n(\%)$ & CHXn (\%) & Total $n(\%)$ \\
\hline Mean age \pm SD (years) & & $19.65 \pm 0.98$ & $19.10 \pm 0.85$ & $19.4 \pm 0.99$ & $19.38 \pm 0.95$ \\
Gender & Males & $12(60)$ & $13(65)$ & $14(70)$ & $39(65)$ \\
& Females & $8(40)$ & $7(35)$ & $6(30)$ & $21(35)$ \\
\hline
\end{tabular}

TTO, tea tree oil; $\mathrm{ClO}_{2}$, chlorine dioxide; $\mathrm{CHX}$, chlorhexidine; $\mathrm{SD}$, standard deviation

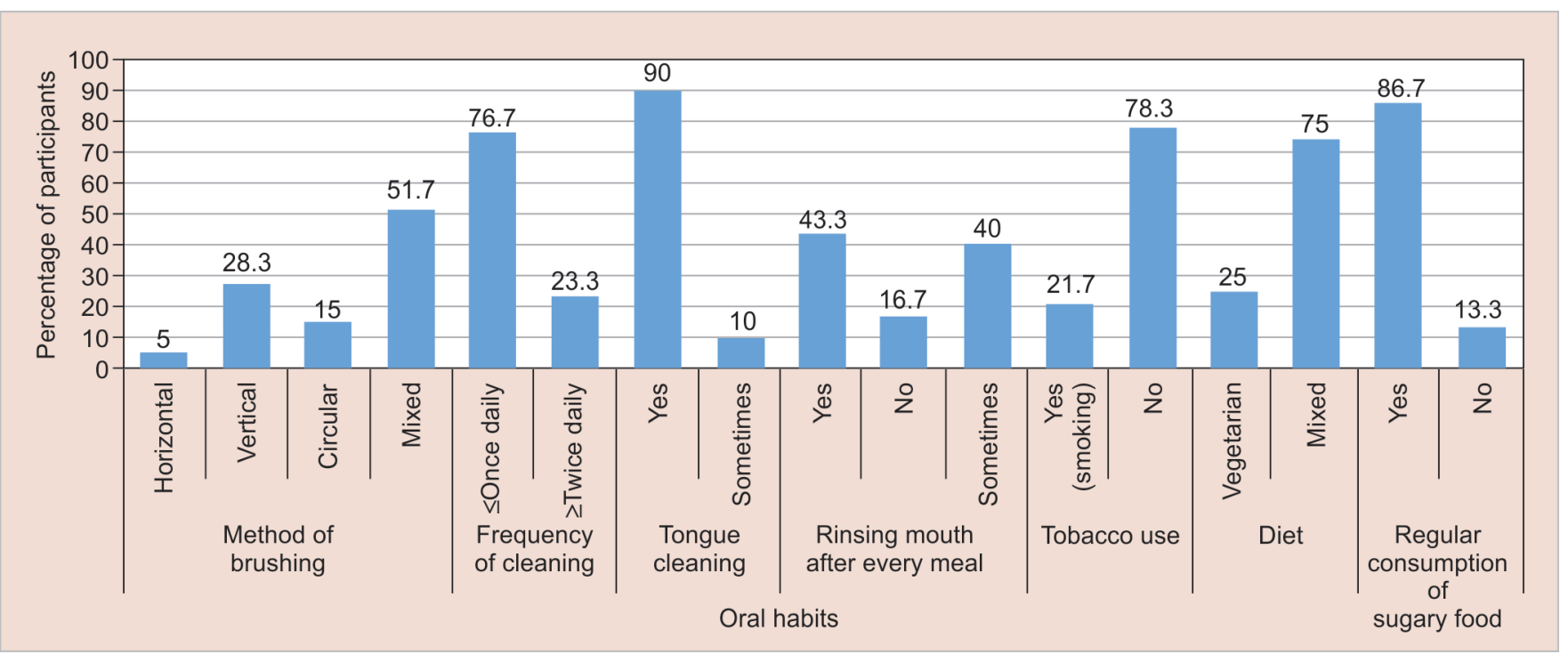

Fig. 1: Distribution of study participants based on their oral health habits

All three types of mouthwash showed a statistically significant reduction in plaque accumulation and gingival inflammation after 21 days (Table 2). However, among the three mouthwashes, TTO mouthwash and chlorhexidine showed a greater reduction in plaque accumulation than chlorine dioxide mouthwash, while chlorhexidine significantly reduced gingival inflammation, greater than the other two types of mouthwash (Table 3).

Figure 2 shows the ratings and feedback of the participants for their respective mouthwashes. While most participants rated all three types of mouthwash good, $5 \%$ of the participants in the chlorine dioxide mouthwash group rated it bad. 
Tea Tree Oil and Chlorine Dioxide Mouthwashes in Reducing Gingivitis

Table 2: Comparison of mean plaque and gingival scores within groups at baseline and follow-up

\begin{tabular}{|c|c|c|c|c|c|}
\hline & Baseline & Follow-up (21 days) & Mean difference & Test statistic & pvalue \\
\hline \multicolumn{6}{|c|}{ Plaque scores ${ }^{a}$} \\
\hline TTO & $1.191 \pm 0.351$ & $0.930 \pm 0.313$ & $0.261 \pm 0.104$ & -3.860 & $<0.001^{*}$ \\
\hline $\mathrm{ClO}_{2}$ & $1.395 \pm 0.345$ & $1.250 \pm 0.337$ & $0.145 \pm 0.094$ & -3.602 & $<0.001^{*}$ \\
\hline $\mathrm{CHX}$ & $1.283 \pm 0.407$ & $0.895 \pm 0.330$ & $0.388 \pm 0.141$ & -3.947 & $<0.001^{*}$ \\
\hline \multicolumn{6}{|c|}{ Gingival scores ${ }^{\mathrm{b}}$} \\
\hline TTO & $1.378 \pm 0.467$ & $1.085 \pm 0.388$ & $0.293 \pm 0.147$ & 8.870 & $<0.001^{*}$ \\
\hline $\mathrm{ClO}_{2}$ & $1.572 \pm 0.342$ & $1.385 \pm 0.331$ & $0.187 \pm 0.101$ & 8.200 & $<0.001^{*}$ \\
\hline $\mathrm{CHX}$ & $1.418 \pm 0.098$ & $0.980 \pm 0.366$ & $0.438 \pm 0.190$ & 10.288 & $<0.001^{*}$ \\
\hline
\end{tabular}

TTO, tea tree oil; $\mathrm{ClO}_{2}$, chlorine dioxide; $\mathrm{CHX}$, chlorhexidine

*Statistically significant at $p<0.05$

ailcoxon rank-sum test,

${ }^{\mathrm{b}}$ paired $t$-test

Table 3: Comparison of mean reduction plaque and gingival scores among groups

\begin{tabular}{|c|c|c|c|c|c|}
\hline Group & $N$ & Mean difference & Test statistic & $p$ value & Post hoc ${ }^{b}$ \\
\hline \multicolumn{6}{|c|}{ Plaque scores ${ }^{a}$} \\
\hline TTO & 20 & $0.261 \pm 0.104$ & 27.429 & $<0.001^{*}$ & TTO vs $\mathrm{ClO}_{2}-0.011^{*}$ \\
\hline $\mathrm{ClO}_{2}$ & 20 & $0.145 \pm 0.094$ & & & $\mathrm{ClO}_{2}$ vs $\mathrm{CHX}-<0.001^{*}$ \\
\hline $\mathrm{CHX}$ & 20 & $0.338 \pm 0.141$ & & & TTO vs CHX -0.063 \\
\hline \multicolumn{6}{|c|}{ Gingival scores ${ }^{a}$} \\
\hline TTO & 20 & $0.305 \pm 0.131$ & 9.803 & $<0.001^{*}$ & TTO vs $\mathrm{ClO}_{2}-0.326$ \\
\hline $\mathrm{ClO}_{2}$ & 20 & $0.227 \pm 0.127$ & & & $\mathrm{ClO}_{2}$ vs $\mathrm{CHX}-<0.001^{*}$ \\
\hline $\mathrm{CHX}$ & 20 & $0.438 \pm 0.190$ & & & TTO vs $\mathrm{CHX}-0.024^{*}$ \\
\hline
\end{tabular}

TTO, tea tree oil; $\mathrm{ClO}_{2}$, chlorine dioxide; $\mathrm{CHX}$, chlorhexidine

*Statistically significant at $p<0.05$

${ }^{a}$ Kruskal-Wallis test

${ }^{\text {b}}$ Dunn's post hoc test

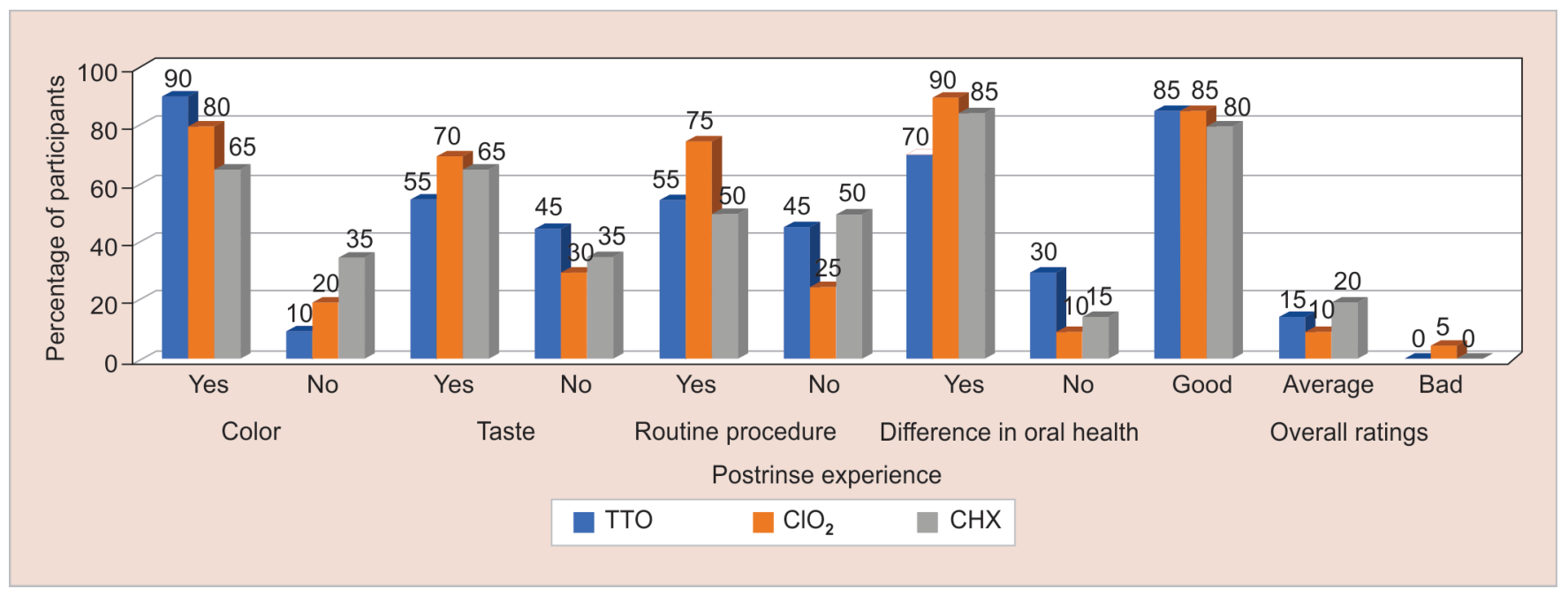

Fig. 2: Distribution of study participants based on their postrinse experience

\section{Discussion}

Gingivitis affects a wide range of the population of all ages and the increase in awareness about oral health has led to an increase in the production of products, synthetic or natural, and their use. With a wide market, dental workers, dentists, and dental hygienists are required to provide information to patients on the selection and use of different dentifrices, with more emphasis on natural products.

The present study was occupied with the effectiveness of TTO and chlorine dioxide mouthwashes on the supragingival plaque growth and gingival inflammation for a period of 21 days using chlorhexidine, the gold standard as a positive control for comparison. The literature has comparisons between the efficacy of TTO or chlorine dioxide mouthwashes with chlorhexidine and/ or placebo rinses.

Daily toothbrushing is the primary and most effective method of plaque removal. The literature shows that the although the individual's ability to brush properly is an important factor, chemical plaque control agents, used adjunctive to mechanical plaque 
control, indeed have a positive effect on gingivitis..$^{13-15}$ To study the real-world effectiveness of these mouthwashes, oral prophylaxis was not performed for the participants at baseline as eliminating calculus and plaque at the beginning of the study can affect the study findings. Instead, they were advised to continue their routine oral hygiene habits. Randomization similarly ensured that all three groups were comparable with regard to their oral habits (hygiene, dietary, and smoking).

In all the three groups in the study, there was a significant decrease in plaque and gingival scores at follow-up compared to baseline, as reported in previous studies. ${ }^{10,16}$ The reduction in gingivitis in TTO group could be attributed to the active compounds-1,8-cineole, terpinene-4-ol, gamma-terpinene, and alpha-terpineol. These compounds also have shown a wide spectrum of antimicrobial and anti-inflammatory actions, ${ }^{5,17}$ thereby causing a reduction in both plaque and gingival scores; ${ }^{10}$ however, Salvatori et al. ${ }^{18}$ observed no difference in plaque scores but significant reduction in gingival inflammation after the use of TTO. Similarly, the antibacterial properties of chlorine dioxide are attributed to its ability in inactivating enzymes, disrupting protein synthesis, oxidizing volatile sulphur compounds, and altering the electrolyte balance within cell membranes, thereby bringing about its lysis. ${ }^{8,19,20}$

The antimicrobial action of chlorhexidine has been well established. Its pin cushion effect damages the surface of microorganisms, causing an osmotic imbalance that leads to precipitation and leakage of cytoplasm, thereby resulting in cell death. ${ }^{21-23}$ With chlorhexidine, within a minute after administration, a reduction of $87 \%$ in aerobes and $84 \%$ in anaerobes is observed, whereas after 5 hours, the reduction of aerobes observed was $88 \%$ and in anaerobes was $92 \% .{ }^{21}$ On comparison with chlorhexidine, TTO showed a similar reduction in plaque scores, as available in the literature ${ }^{10}$ while being significantly less than chlorhexidine regarding the reduction of gingival scores. ${ }^{10}$ Reduction of plaque accumulation by chlorine dioxide was significantly less than chlorhexidine as observed in other studies. ${ }^{7}$ However, contrasting results are available in the literature as well. ${ }^{16} \mathrm{~A}$ Cochrane review in $2017^{24}$ concluded that in individuals with moderate or severe levels of gingivitis, there was insufficient evidence to determine if reduction in gingivitis was associated with chlorhexidine mouth rinse use. In this study, all three mouthwashes, including chlorhexidine, showed a significant reduction in gingivitis. The properties of substantivity and pin cushion effect are what make chlorhexidine the gold standard. While the two mouthwashes in this study do not possess either of these two properties and are not as effective as chlorhexidine in reducing gingivitis, they however did significantly reduce plaque accumulation and gingivitis in moderate to severe gingivitis. These results imply that more research is required with respect to the patterns and effects of its long-term use as compared to those of chlorhexidine.

Tea tree oil can have side effects such as toxicity if ingested in higher doses, skin irritation, allergic reactions, etc. ${ }^{17}$ Chlorine dioxide similarly has not shown any significant side effects. ${ }^{25}$ However, longitudinal studies are needed to study the long-term effects of these mouthwashes. Regarding the side effects of mouthwashes in this study, $45 \%$ reported bitter taste with TTO as compared to $30 \%$ in the chlorine dioxide group. 30\% Overall, TTO and chlorine dioxide were preferred and rated good by most participants.

Although this study assesses the effectiveness of TTO and chlorine dioxide by comparing it to chlorhexidine, it does not address the possible long-term outcomes of both the mouthwashes and supervision of regular usage of mouthwash could not be monitored. The study also included young adult population as higher education background makes it easier for them to understand the concept of prevention and well-being, along with proper utilization of the dental-care services. Thus, studies are required to assess its acceptability and effectiveness among adults. Despite the limitations, susceptibility of oral microorganisms to TTO and chlorine dioxide has been demonstrated, indicating that incorporating it into appropriate mouthwashes would be beneficial.

\section{Conclusion}

The study concluded that all three types of mouthwashes were effective as antiplaque and antigingivitis agents. On comparison with chlorhexidine, TTO showed similar reduction only in plaque accumulation while chlorine dioxide was not as effective. Nonetheless, both TTO-based mouthwash and chlorine dioxide mouthwash can be used to reduce plaque and gingivitis.

\section{ACKNOWLedgments}

The authors are grateful to the study participants for their cooperation in the study, Dr Khechari Chavan for her assistance in data acquisition and Dr Kamala D. N., Group Pharmaceuticals Ltd., for her support.

\section{Manufacturer Name}

Guard-OR, Group Pharmaceuticals Ltd, Bengaluru, India Freshchlor, Group Pharmaceuticals Ltd, Bengaluru, India NATURALIS: Essence of Nature Tea tree essential oil, Bengaluru, India

\section{References}

1. Chandki R, Banthia P, Banthia R. Biofilms: a microbial home. J Indian Soc Periodontol 2011;15(2):111-114. DOI: 10.4103/0972-124X.84377.

2. Preshaw PM. Detection and diagnosis of periodontal conditions amenable to prevention. BMC Oral Health 2015;15(S1):S5. DOI: 10.1186/1472-6831-15-S1-S5.

3. Haerian-Ardakani A, Rezaei M, Talebi-Ardakani M, et al. Comparison of antimicrobial effects of three different mouthwashes. Iran J Public Health 2015;44:997-1003.

4. Safiaghdam H, Oveissi V, Bahramsoltani R, et al. Medicinal plants for gingivitis: a review of clinical trials. Iran J Basic Med Sci 2018;21:978991.

5. Carson CF, Riley TV. Antimicrobial activity of the major components of the essential oil of Melaleuca alternifolia. J Appl Bacteriol 1995;78(3):264-269. DOI: 10.1111/j.1365-2672.1995.tb05025.x.

6. May J, Chan $\mathrm{CH}$, King A, et al. Time-kill studies of tea tree oils on clinical isolates. J Antimicrob Chemother 2000;45(5):639-643. DOI: 10.1093/jac/45.5.639.

7. Paraskevas $S$, Rosema NA, Versteeg $P$, et al. Chlorine dioxide and chlorhexidine mouth rinse compared in a 3-day plaque accumulation model. J Periodontol 2008;79(8):1395-1400. DOI: 10.1902/jop.2008.070630.

8. Shinada K, Ueno M, Konishi C, et al. Effects of a mouthwash with chlorine dioxide on oral malodor and salivary bacteria: a randomized placebo-controlled 7-day trial. Trials 2010;11:14. DOI: 10.1186/17456215-11-14.

9. Rath SK, Singh M. Comparative clinical and microbiological efficacy of mouthwashes containing $0.2 \%$ and $0.12 \%$ chlorhexidine. Dent Res J (Isfahan) 2013;10:364-369. 
10. Kamath NP, Tandon S, Nayak R, et al. The effect of aloe vera and tea tree oil mouthwashes on the oral health of school children. Eur Arch Paediatr Dent 2020;21(1):61-66. DOI: 10.1007/s40368-019-00445-5.

11. Silness J, Loe H. Periodontal disease in children, a correlation between oral hygiene and periodontal condition. Acta Odontol Scand 1964;22(1):121-135. DOI: 10.3109/00016356408993968.

12. Loe $\mathrm{H}$, Silness J. Periodontal disease in pregnancy 1: prevalence and severity. Acta Odontol Scand 1967;21(6):533-551. DOI: 10.3109/00016356309011240.

13. Claydon NC. Current concepts in toothbrushing and interdental cleaning. Periodontol 2000 2008;48(1):10-22. DOI: 10.1111/j.16000757.2008.00273.x.

14. Figuero E, Nóbrega DF, García-Gargallo M, et al. Mechanical and chemical plaque control in the simultaneous management of gingivitis and caries: a systematic review. J Clin Periodontol 2017;44:S116-S134. DOI: 10.1111/jcpe.12674.

15. Teles RP, Teles FRF. Antimicrobial agents used in the control of periodontal biofilms:effective adjuncts to mechanical plaque control. Braz Oral Res 2009;23(suppl 1):S39-S48. DOI: 10.1590/S180683242009000500007.

16. Yeturu SK, Acharya S, Urala AS, et al. Effect of Aloe vera, chlorine dioxide, and chlorhexidine mouth rinses on plaque and gingivitis: a randomized controlled trial. J Oral Biol Craniofac Res 2016;6(1):54-58. DOI: 10.1016/j.jobcr.2015.08.008.

17. Hammer KA, Dry L, Johnson $M$, et al. Susceptibility of oral bacteria to Melaleuca alternifolia (tea tree) oil in vitro. Oral Microbiol Immunol 2003;18(6):389-392. DOI: 10.1046/j.0902-0055.2003.00105.x.

18. Salvatori C, Barchi L, Guzzo F, et al. Comparative study of antibacterial and anti-inflammatory effects of mouth rinse containing tea tree oil. Oral Implantol (Rome) 2017;10(1):59-70. DOI: 10.11138/ orl/2017.10.1.059.

19. Yates R, Moran J, Addy M, et al. The comparative effect of acidified sodium chlorite and chlorhexidine mouth rinses on plaque regrowth and salivary bacterial counts. J Clin Periodontol 1997;24(9):603-609. DOI: 10.1111/j.1600-051X.1997.tb00236.x.

20. Al-bayaty F, Taiyeb-ali T, Abdulla MA, et al. Antibacterial effect of chlorine dioxide and hyaluronate on dental biofilm. Afr J Microbiol Res 2010;4:1525-1531.

21. Eaton KA, Rimini FM, Zak E, et al. The effects of a $0.12 \%$ chlorhexidine digluconate-containing mouth rinse versus placebo on plaque and gingival inflammation over a 3-month period: a multicentre study carried out in general dental practices. J Clin Periodontol 1997;24(3):189-197. DOI: 10.1111/j.1600-051X.1997.tb00490.x.

22. Van Strydonck DA, Slot DE, Van der Velden U, et al. Effect of a chlorhexidine mouth rinse on the plaque, gingival inflammation and staining in gingivitis patients: a systematic review. J Clin Periodontol 2012;39(11):1042-1055. DOI: 10.1111/j.1600-051X.2012.01883.x.

23. McBain AJ, Bartolo RG, Catrenich CE, et al. Effects of a chlorhexidine gluconate-containing mouthwash on the vitality and antimicrobial susceptibility of in vitro oral bacterial ecosystems. Appl Environ Microbiol 2003;69(8):4770-4776. DOI: 10.1128/AEM.69.8.47704776.2003.

24. James $\mathrm{P}$, Worthington HV, Parnell C, et al. Chlorhexidine mouthrinse as an adjunctive treatment for gingival health. Cochrane Database Syst Rev 2017;3(3):CD008676. DOI: 10.1002/14651858.CD008676.pub2.

25. Ratcliff JL, Renken EA, Ward JK, Method for determining the effectiveness of stabilized chlorine dioxide in a mouth rinse. US Patent No. US7875460B2. 2011. 